\title{
Identifying Evacuation Assembly Points: An Application at Latsia, Nicosia, Cyprus
}

\author{
Savvas Chrysoulidis ${ }^{1}$
}

This article is based on a presentation delivered at the $4^{\text {th }}$ International Academic Conference on Human Security, Belgrade, organized by the Faculty of Security Studies, University of Belgrade, 2-3 November 2018. The author is grateful for the feedback received during the conference.

\section{Abstract}

The occurrence and magnitude of real-world threats and challenges are difficult to predict in time and space, as they often cause new, unexpected situations, making them extremely difficult to manage. The present paper explores how applied human security could allow for a coordinated and secure allocation of population in emergencies. The use of new technologies could provide capabilities and tools for policy/decision-makers and first responders in order to facilitate such allocation endeavours. Cases demanding an organized evacuation in urban areas (large or small) are when disasters occur or are estimated as highly probable to happen in the near future. The identification of proper Emergency Assembly Points - EAPs plays a significant role in almost all crisis and disaster management situations, particularly in efficient and effective planning and responding.

Keywords: applied human security, geospatial profile, terrain analysis, disaster management, GIS

1 Savvas Chrysoulidis, Ph.D. Candidate, Department of Civil Engineering and Geomatics, Cyprus University of Technology (CUT), Greece, 0030 6972932936, E-mail: se.chrysoulidis@edu. cut.ac.cy

Issue 3 / 2019

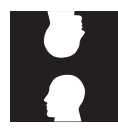




\section{Identifying Evacuation Assembly Points: An Application at Latsia, Nicosia, Cyprus}

\section{Introduction}

Sustainable development is intimately connected to human security, one dimension of which is the risk due to natural disasters. At the same time, human interventions in the environment, be it at the scale of individuals, communities, or nations, can enhance the existing or generate new disaster risks. On the contrary, it contributes to a significant reduction in disaster risk. Considering security stakeholders, policy/decision-makers, and especially first responders in the field, this poses a real challenge in terms of security provision. Compensatory disaster risk and crisis management, such as disaster preparedness, civil-society resilience, and response stand alongside development planning. Furthermore, it focuses on the amelioration of existing vulnerability and the reduction of natural hazards that have accumulated through past development pathways. Compensatory policy is necessary to reduce contemporary risk. At the same time, the prospective system is required for medium- to long-term disaster risk reduction (UNDP, 2004). In this context, several decisions and preparations about how to inform, allocate, and organize the movement of possibly thousands of people should be made. The responsibility for such choices remains with the State: "Governmental security stakeholders retain the primary role and responsibility to protect their citizens" (United Nations General Assembly, 2012).

Until now, governmental (public) security stakeholders have considered/treated individuals as a collective, unified, and indivisible whole, termed society or community. This approach has a negative impact on people's security 'mentality' which reflects their roles and responsibilities from minor to significant security incidents. Moreover, in the context of planning against crises/emergencies, individuals most often have a neutral or negative 


\section{European Human \\ Journal of Security}

Page I 50

participation attitude. This attitude leads stakeholders responsible for policymaking, or even first responders, to consider individuals more as victims than as equally capable stakeholders in terms of emergency response (Chrysoulidis \& Kyriakidis, 2018).

Additionally, it is imperative to highlight the fact that except for the direct benefits of protecting human lives by providing safe areas, there is another essential impact to be exerted by the organized and informed/prepared individuals beyond evacuation plans in pre-determined areas. This positive impact is connected to enhancing individuals' security mentality and social resilience. Both are vital at all levels of stakeholders, from individuals to State, to overcome all types of crises and emergency incidents. The main complication of planning begins with human nature. People tend to act habitually and in stable ways over time. Additionally, there is a strong possibility that they may not even react when circumstances arise, even when their own life is directly under threat. Last but not least, new technologies play an important role and, in particular, may facilitate individuals, even in an unconscious way, to become more active and obtain or increase their security mentality that includes all safety and security issues.

The above implies that authorities of public security should start considering and involving individuals in the planning process. A very first step in this direction could be to inform them about the variety of threats that might occur and endanger them based on their location. In that direction, authorities can indicate to individuals the pre-chosen EAPs (safe areas), where during ongoing emergencies population has to move on, usually only after responsible authorities have asked/informed them to go. Furthermore, following specific procedures and taking into account individuals' particular needs can construct a useful tool/method which will create/enhance the security mentality of the population. For society, the aftereffect of improving a specific mindset of security is the development of social security culture (CPNI, 2018). Also, it increases social resilience and translates it into an efficient and effective response of a society to 
emergencies. The new technologies, like Geographic Information System - GIS and remote sensing combined with GIS, are some of the many technologies that could be used to strengthen further individual mentality and physical security. Independently of how people will evacuate the affected area, the primary consideration remains where these people should resort to and be safe. The identification of appropriate secure areas relates both to physical geographical factors (terrain analysis) and to human geographical factors (population characteristics). Both physical and human environment characteristics comprise the Geospatial Profile (see below).

A significant issue in population allocation during emergencies is the identification and determination of safe areas/destinations, named Emergency Assembly Points (EAPs). Different factors and criteria should be taken into consideration by the public security stakeholders, who are responsible for planning and executing the evacuation of the population. For instance, depending on whether they possess a car or not, people from the affected regions may evacuate the area by vehicle, foot, or other public means (provided by the authorities). Nevertheless, we should be careful if there are some individuals or groups of people who are not able to evacuate on their own (e.g. patients in hospitals, older people, people with disabilities, children in school hours, and others). Special provision regarding the use of transportation means or ambulances is needed, it is vital to evacuate these vulnerable population categories out of dangerous areas. In urban areas, it can be assumed that evacuation by vehicle will be the leading choice of most of the people who will decide to evacuate themselves during or before an emergency occurs.

In the next section, the concept of applied human security is introduced, along with the framework in which the proposed methodology for EAP identification has been developed. This second section also presents the necessary definitions and concepts regarding both physical and human environment. The third section offers the data used to illustrate the application of the proposed 


\section{European Human}

methodology regarding EAP identifications. The fourth section presents the final results, showing the selected EAPs in map format. The last part recommends the actions that should be taken by state security stakeholders and concludes presenting the benefits of new technologies in human security that could be enhanced in combination with the use of personalized human security concepts.

\section{Methodology}

Security authorities/stakeholders at all levels have the responsibility/duty to respond to the rapidly and continuously changing operating environment, to provide societies with the public good of security. They are called on to play exigent multidimensional roles. Their ultimate mission is to ensure the lives of citizens, the establishment of citizens' security feeling, and proper management of available resources to succeed in their mission. Demanding roles which they are called to respond to involve them in diverse and complex fields (urban/residential areas, forests, rocky-mountains, marine areas), emergencies/situations which they have to accomplish/deal with, hazards/natural disasters (fires, floods, earthquakes, tsunamis), internal security issues (asymmetric threats - terrorism, irregular/mass refugees flow), man-made disasters (industrial accidents, mature pollution - degradation). These are only some of the many unexpected situations that they have to respond to effectively and efficiently, accomplishing their missions. For their success against these challenges and threats, it is necessary to use/integrate new technologies with the benefits resulting from their use. It will allow security stakeholders to achieve efficient and timely decision-making processes in the planning phase and ongoing emergencies/issues by identifying proper EAPs.

Security stakeholders consider contemporary urban environments as multidimensional and complex. Within an emergency response context, complex environments require extensive understanding, analysis, and continuous situation assessment. Security safeguards should be able 


\section{European Human}

to determine the identity, socio-economic status, cultural background, and other elements of individuals and groups which might be at risk due to potential threats. New technologies, such as Geographic Information Systems - GIS, remote sensing, and geospatial data analytics, provide the required toolbox. They provide capabilities for the identification and analysis/modelling manners/procedures that should be considered during the evacuation into Emergency Assembly Points. Applied human security implements new technologies' capabilities combined with theoretical knowledge.

Up-to-date bibliographies regarding security studies approach the issue of human security by focusing mostly on theoretical analysis, and, second, on how the applications can enhance it. The interest of the researches mainly lies in how and by which factors human lives are influenced and threatened. Regarding the fact that there are numerous HS definitions and approaches, we can conclude that the concept of human security is concerned with both approaches, theoretical and academic. Also, it indicates the need for a deeper approach to this issue, grounded in practice and covering equally the governmental policydecision making authorities and first responder. In order to protect human lives effectively, security stakeholders must deliberately identify and prepare for the distinct threats.

Moreover, it should be taken into consideration that every threat has unique characteristics relevant to the location where it occurs which are changing (speedily) over time. The fact is that even when the same danger arises in the same area, it has different impacts over time. It becomes easily understandable that EAP is perhaps the only benchmark that provides a stable base to start with the crisis management planning and enhance human security.

The chosen applied methodology in the current paper, primarily based on physical terrain analysis and, second, on human terrain analysis, in an order which is based on specific criteria to identify the proposed/selected areas. Identified/selected areas must offer safety and security combined with a well-established road network. The 


\section{European Human}

chosen locations must have more than one exit and entry points/routes. Additionally, the chosen areas must have a suitable size according to the evacuated population. Also, the selected areas should identify the spots/locations which are vulnerable to other potential threats (like dams, forests) and characterize them as unsuitable for sheltering people in case of evacuation. The essential first step is to take into consideration equally physical and human characteristics, regarding both area/location and population, and finally to succeed and enhance security provision.

\section{Physical Environment}

Impacts of physical environment originate from studies on the vulnerability (Lukacs \& Bhadra, 2012) and capacities (Hansford, 2011) of individuals towards natural hazards (Papathoma-Köhle et al., 2007) and other environment-based emergencies. There are specific physical environment factors that influence the way and the level of a person or group of people who are affected (by reducing or increasing the individual's capacities and vulnerabilities). Factors, namely, are the precise position details of that person or group of people - Coordinates, Elevation, Slope, Hydrology of the Area, Land Cover Land Use, and others. Physical terrain differentiates from place to place, as well as over time. Identification of suitable EAPs has to follow specific chosen criteria. Physical terrain characteristics, in combination with human terrain analysis and features, will allow us to identify the desired proper areas and use them as EAPs. Methodology will be applied based on the process and merging of different layers and datasets from variable sources, as below:

CORINE Land Cover/Use. CORINE Land Cover (CLC) inventory was first used in 1985 (reference year 1990). It is a land cover classification system that is composed of a variety of applications, underpinning various community policies in many different domains of the environment. Also, in other fields, e.g. agriculture, transport, spatial planning, safety, security, and others. Several updates were 


\section{European Human}

produced in 2000, 2006, 2012, and 2018 (CORINE Land Cover, 2018). It consists of an inventory of land cover in 44 classes. CLC uses a Minimum Mapping Unit (MMU) of 25 hectares (ha) for areal phenomena and a minimum width of $100 \mathrm{~m}$ for linear phenomena. CLC is a 3-level hierarchical classification system and has 44 classes. The third level is the most detailed level. There is an extra class that is not included in 44 classes, one of no data. In the present paper's study area, only 11 out of the total 44 CLC classes are identified. CLC is one of the layers which will be used to detect impacts (negative or positive) regarding both land cover and sheltering of the population in an evacuation. CLC could make it impossible and even dangerous to use an area because the land cover/use type may NOT provide the proper conditions.

Digital Elevation Model - DEM. Advanced Spaceborne Thermal Emission and Reflection Radiometer (ASTER) Global Digital Elevation Model Version 3 (GDEM 003) can be found and downloaded as open-source data, with free license policy (ASTER Global Digital Elevation Map Announcement, 2004). DEM can be used to determine terrain attributes such as elevation at any point (pixel), slope, and aspect. Today, most GIS applications depend mainly on DEM. By processing DEM, we can find the size of the selected area in combination with its slope and altitude. Moreover, we can create the hydrographic network (a total of water bodies and streams in an area - rivers, lakes, swamps, and water reservoirs), which plays an essential role in selecting an EAP. It is critical to choose an area with proper slope and altitude that will offer the desired protection to the evacuated population. Otherwise, for instance, in case of an ongoing flood or heavy rain in combination with proximity to a neighbour stream, other potential dangers can threaten the evacuated population. Many different factors should be taken into authorities' consideration regarding DEM. For example, in case of shelter establishment after an earthquake, the slope should not be too steep, as it may cause problems in establishing the necessary facilities. 


\section{European Human \\ Journal of Security}

\section{Human Environment}

Human environmental factors that are connected to our research arise mainly from the answer to the question which factors affect the individual in the decision-making process; We should focus on the behaviour of a person or group of people during crises and other threatening socio-economic phenomena (Arru \& Negre, 2017). Categories of human-environment elements are: socioeconomic status (SES) (age, sex, nationality, religion, residence place, level of education, civil status (married with children, divorced), income (wealthy, car owner) and occupation), health condition, government or other role (civilian/civil protection/local or federal enforcement/ Armed Forces). Additionally, all man-made structures are considered as part of the human-environment even if they are in direct connection to physical environment features, e.g. road networks, which play a highly significant role in our methodological approach and analysis below.

Open Street Map - OSM. Open Street Map is an opensource, free, editable map of the whole world that is being built by volunteers (OpenStreetMap, 2006). A road network is an essential factor for the final identification of suitable areas for EAPs. It is crucial not only for the classification of the roads which provide the necessary access to the EAPs, but also for the precise calculation of the number of routes in the specific area. Ideally, more than one entry and exit route/point is a minimum factor for designating an area as an EAP.

\section{Geospatial Profile}

The necessity for using new technologies in the security domain which are specific to human security relates to the current environment where security stakeholders have to act/operate. Moreover, an equally important factor is the fact that classical approaches/solutions to modern threats/problems seem to be incompetent in responding in an accurate and timely manner. This failure is recorded due to the lack of analysis of the natural environment in close interaction with the human environment. 


\section{European Human \\ Journal of Security}

Furthermore, in current approaches, researchers do not take into consideration the dynamic evolution of natural phenomena. These phenomena are always changing based on many rapidly evolving variables (complex environment). Also, it is vital to deal with all modern threats in combination with the unique geospatial profile of each individual. Geospatial profile (spatial identity) (Figure 1), refers to the characteristics/elements of each person, based on two pillars comprising the physical (natural) and human environment (Chrysoulidis \& Kyriakidis, 2018).

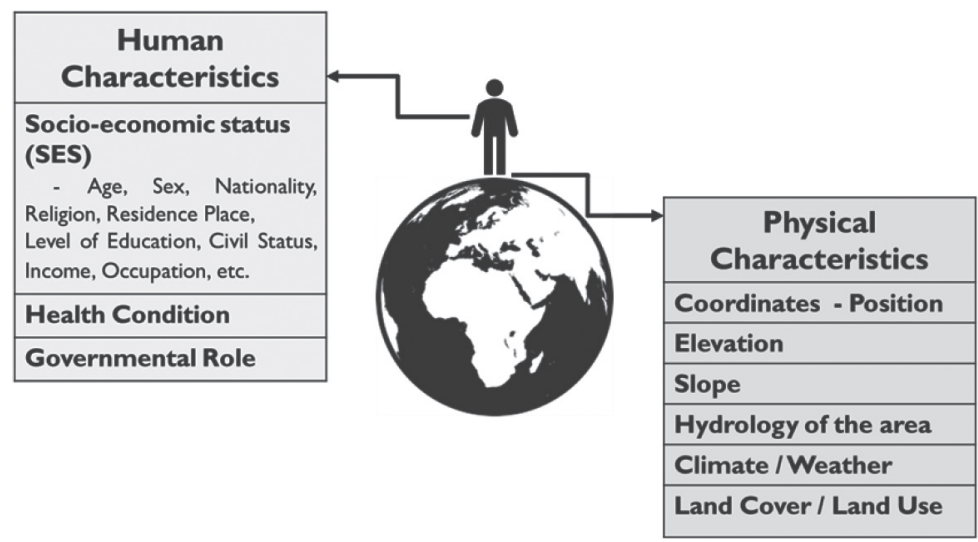

Figure 1: Geospatial profile's constituent elements

Geospatial profile is dynamic because its constituent elements change continuously over time (short- and long-term). Also, the geospatial profile is person-specific since its constituent elements are unique for each person. Furthermore, the spatiotemporal 'identity' of each human being can be considered. Geospatial profile enables us to combine equally human and physical terrain in order to succeed in a holistic approach in the whole process regarding the population evacuation. Not only by identifying the most suitable areas (physical dimension), but also by determining how the population can be prepared to successfully and safely relocate to EAPs.

Moreover, under some parameterization of the constituent elements, the geospatial profile could be used in an aggregated or mean sense to characterize 


\section{European Human \\ Journal of Security}

Page I 58

groups of people, organisations, communities, regions, and generally well-defined structures with spatial characteristics. Aftereffect of that capability at the local level of municipalities (where all the necessary data already exists) makes it feasible to inform all the residents personally, about where and how they should arrive at EAPs. In this procedure, by using the geospatial profile, authorities are forced to consider all the unique personal characteristics of each person for the planning and response phases.

\section{Data}

ArcGIS ESRI (ArcMap 10.7) software is being used in the present paper for the processing of the selected datasets and construction of the desired data layers. ESRI's software ArcMap offers the users the capability to use specific tools for the desired geographic transformations of the data. That means - to translate coordinates from one geographic coordinate system to another. When the user adds a layer to a map with a different geographic coordinate system, a warning from software comes up, and the change to the coordinate system is applied automatically. Furthermore, the user can define the data with the proper and desired coordinate system, which is the same for all the selected data layers. In our study, in order to succeed, we used higher accuracy and highquality data and worked on a projected coordinate system. Projection is a mathematical transformation that takes spherical coordinates and transforms them into an XY (planar) coordinate system. Projections are determined based on the needs of the map or data analysis and the area of the world. In this paper, the chosen study area is in Cyprus, where the projected coordinate system is the WGS 84 / UTM zone 36N. That is suitable for use between $30^{\circ} \mathrm{E}$ and $36^{\circ} \mathrm{E}$, northern hemisphere between Equator and $84^{\circ} \mathrm{N}$, onshore and offshore. The states that are suitable for this projected coordinated system are Belarus, Cyprus, Egypt, Ethiopia, Finland, Israel, Jordan, Kenya, Lebanon, Moldova, Norway, and the Russian Federation. The WGS 84 / UTM zone 36N uses the WGS 84 


\section{European Human \\ Journal of Security}

geographic 2D Coordinate Reference System - CRS as its base CRS and the UTM zone 36N (Transverse Mercator) as its projection. The WGS 84 / UTM zone $36 \mathrm{~N}$ is a CRS for large- and medium-scale topographic mapping and engineering survey.

Forsmall-scaleevacuations (buildings, neighbourhood) inside the residential areas, open spaces/areas are used 59 I Page as assembly points. These areas are in proximity to the incident and always inside the boundaries of the urban area. As a result, these areas are mainly parks, stadiums, public open fields, and others. All these points are suitable to evacuate a small number of people in case of minor, limited in size incidents with limited impact only on a specific and small number of individuals. In this paper, we focus on mass populations' evacuations, considering how we can evacuate an entire town or city.

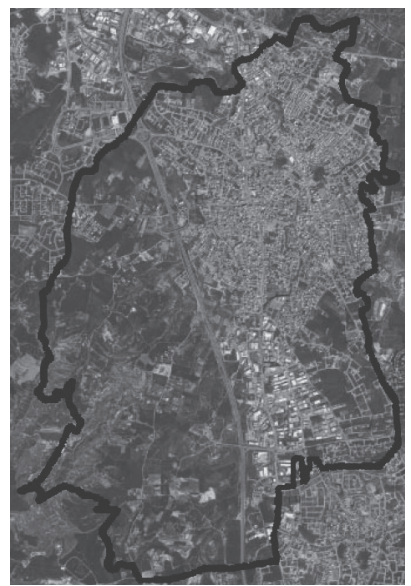

Figure 2: Latsia municipality bounds

The study area is Latsia municipality (fig. 2), and our research task is to identify suitable EAPs inside the municipality's boundaries. The use of well-determined boundaries, such as the political boundaries of the municipality, allows us to standardize the method of evacuation area. The identification is based on the recognized political bounds avoiding overlapping EAPs. Moreover, it offers the methodology of a reference starting point. In case of the absence of proper areas found on our predetermined criteria, we could search for suitable places out of the specific municipality's bounds in another neighbouring municipality. In both situations (small or large evacuation, in or out of residential area), the ultimate task remains to prevent the loss of human lives and people injuries. Extraordinary or preventive evacuation is organised, it could be described as a complex process involving actions 


\section{European Human \\ Journal of Security}

for rapid and safe removal of people to secure areas, as far as possible from the threat (Southworth, 1991).

Corine Land Cover - CLC in the selected study area (Fig. 3) involved 11 land cover categories from the total 44 that CLC used to categorise the different types of land cover.
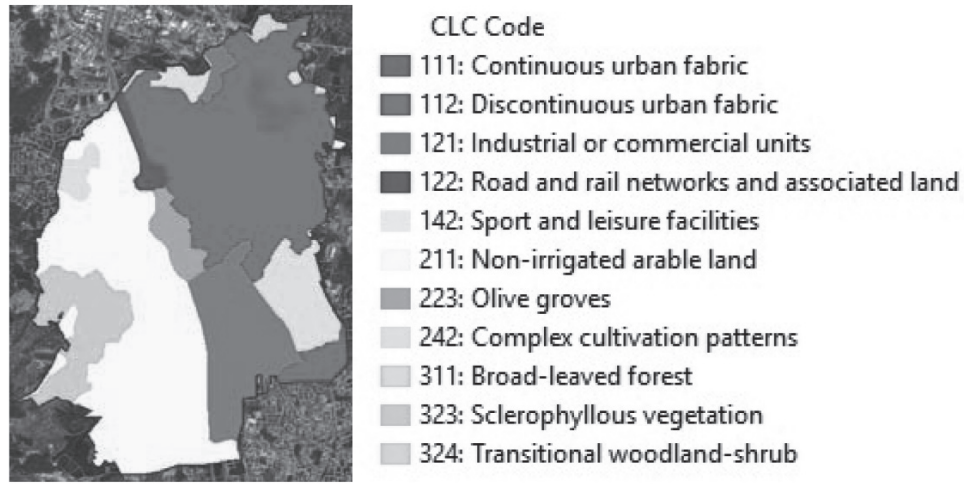

Figure 3: Latsia municipality CORINE Land Cover with 3-digit codes

The following is to identify which of these 11 types of land cover/use are suitable to be chosen as suitable potential EAPs and which are not. Based on Figure 3 categorization, categories 111,112,121, 122 and 142 are not ideal as they belong to residential areas or contain continuous human-made structures. Classes with codes $223,242,311,323$ and 324 are rejected because of the type of vegetation (could be chosen in case no other proper area can be found), which covers those areas and prevents the gathering of the population. Code 211 is recorded as the non-irrigated arable land, and it is the preferable type of land cover. This type/class of CLC offers the optimum characteristics for an evacuation area regarding the land cover and use. We could easily understand that the choice of the proper area depends on the analysis criteria. These are different for every situation, depending on risk analysis factors and potential threats that are different in every place and time.

Following the process of the Digital Elevation Model - DEM (Fig.4), the areas to be rejected were identified the hydrographic network with its streams. Depending on the stream order (stream order based on the Strahler 


\section{European Human}

method), it plays a negative role combined with most potential threats. Moreover, the population should avoid being in proximity to streams, rivers, and generally water bodies. For these reasons, we have to reject areas where rivers or streams have been identified. Additionally, we should add a buffer zone around the water bodies farther from their bank-full width up to topographic floodplain. By making these extensions, we ensure the avoidance of the threats from potential future floods. Selections of streams will follow the highest order following Strahler streams ordering. This order is one of the methods for classifying the hierarchy of natural channels in a watershed (Strahler, 1957). DEM, by the proper process, allows the construction of slope and altitude maps (fig. 4 ). Both the slope and altitude should be under our consideration in the whole process as each situation that can affect our decision about the selection of proper EAPs depends on them.

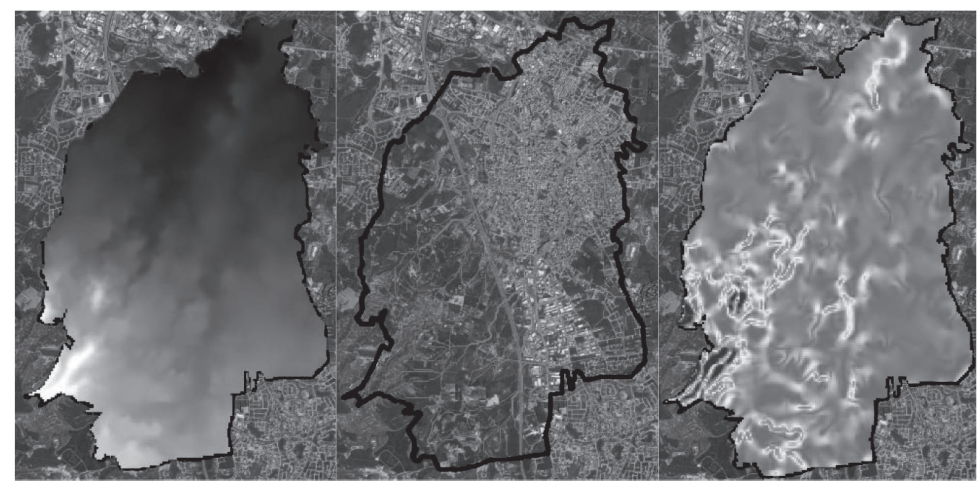

Figure 4: Latsia municipality DEM, hydrographic network and slope maps

The last step for the identification of proper EAPs is to allocate equal population to the selected areas. Method for an equal division of the population is based on Thiessen polygons, a tool of ArcGIS software. This tool is used to divide an area covered by the input point features (in our case, the selected EAPs as point features and Latsia municipality residential area as the selected area for the division) into Thiessen or proximal zones. These zones represent full areas where any location 
within the zone is closer to its associated input point than to any other input point. By using the Thiessen tool/ method we can succeed in dividing the residential area equally, based on the proximity to the areas selected as EAPs. A disadvantage to this approach is the possibility for a building to belong to two different areas. For this reason, it is necessary, after the use of the tool researcher, to check the area and provide the necessary corrections manually. By overlapping, the created layer with the road network makes it possible to connect the divided areas with the roads (Fig. 5), which the population has to follow to arrive safely at specific EAPs.
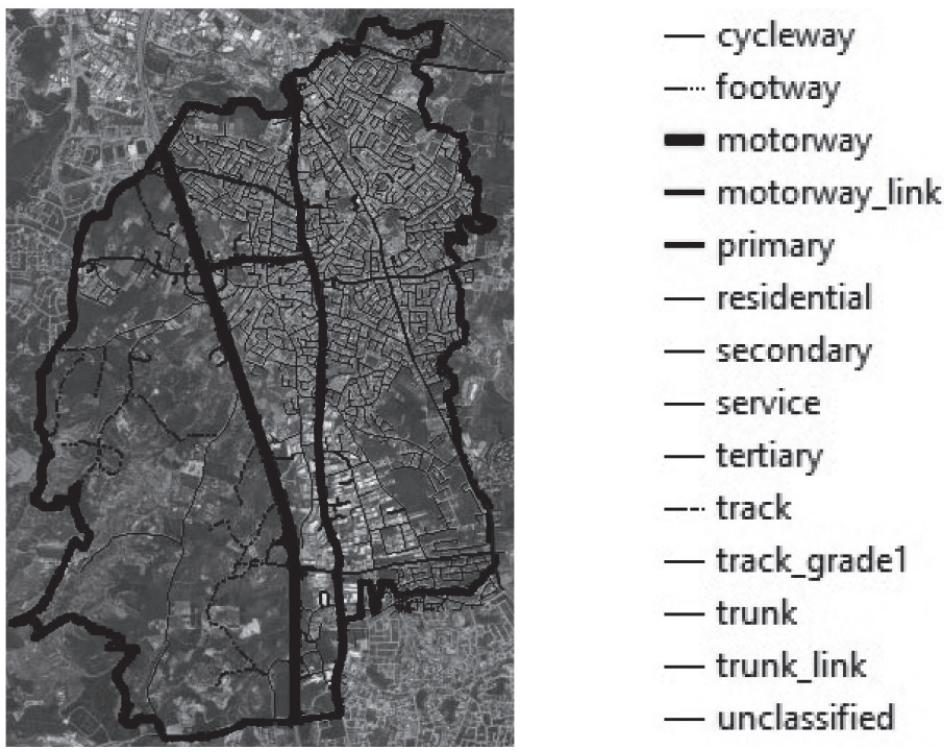

Figure 5: Latsia municipality road network, categorization form OSM 


\section{Results}

\section{Corine Land Cover}

Processing Corine Land Cover datasets will result in the creation of the final CLC map. The final map allows us to identify proper areas for EAPs (fig. 6).

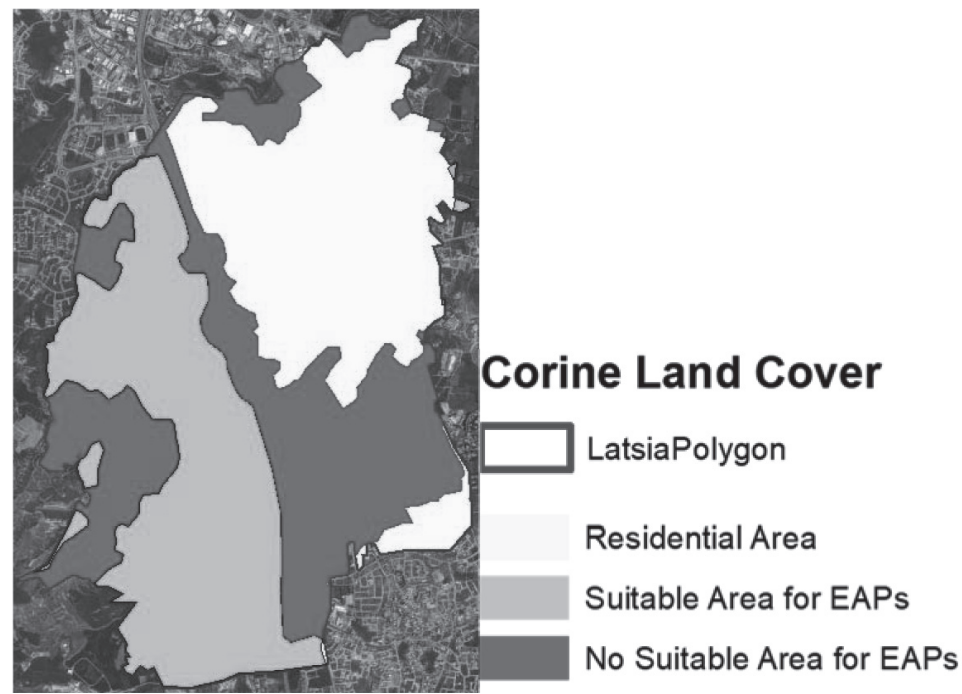

Figure 6: Latsia Corine Land Cover Map with suitable areas for EAPs

\section{Digital Elevation Model}

DEM's process creates the desired datasets of the Latsia area, slope, and hydrology maps.

Slope Map. A slope map can be produced by using the ArcMap toolbox, the proper tool following a specific order/path (ArcToolbox $\rightarrow$ Spatial Analyst Tools $\rightarrow$ Surface $\rightarrow$ Slope). DEM format named raster consists of pixels. We choose between percentages or degrees $(100 \%=45$ degrees). With that order, we succeed in transforming the altitude map to the slope map (Fig. 7) in raster format. In our method, slope plays an important role. Areas with a slope of over $6 \%$ do not indicate them as normal vehicle mobility areas (American Association of State Highway and Transportation Officials, 2001). 


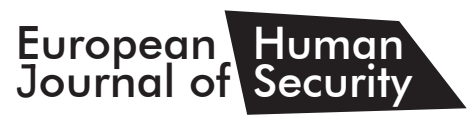

Page I 64

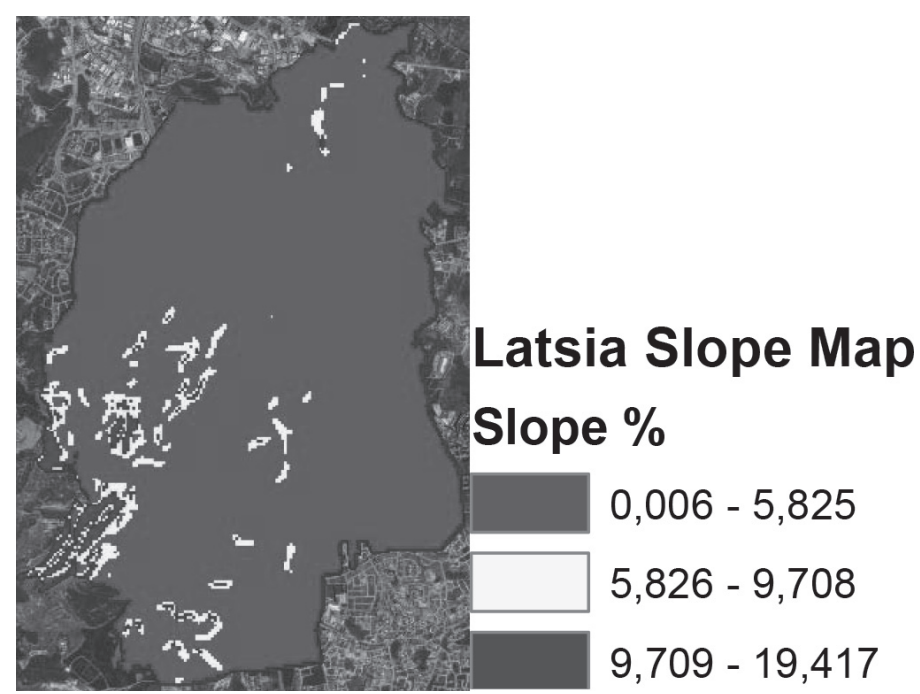

Figure 7: Latsia slope map with a suitable slope for EAPS

Hydrological Map. Hydrological map is produced following the Strahler stream order method. Stream order is a method of setting a numeric order to connect them in a stream network. Strahler stream order is a method that allows us to identify and classify types of streams based on their number of tributaries. Regarding this method, when two first-order streams unite (come together), they form a new second-order stream. The same occurs for the next orders when two second-order streams come together, they form a third-order stream. Latsia's area belongs to the sixth-order stream area. In Figure 8, after the processes are illustrated, streams only from the third to sixth stream orders are included. The first and second stream orders are deleted because they do not influence the terrain to be used as EAPs. The surface, which is covered by the third to sixth stream orders and $10 \mathrm{~m}$ around the streams (fig. 8), will be identified as a non-suitable area for EAP. 


\section{European Human Journal of Security}

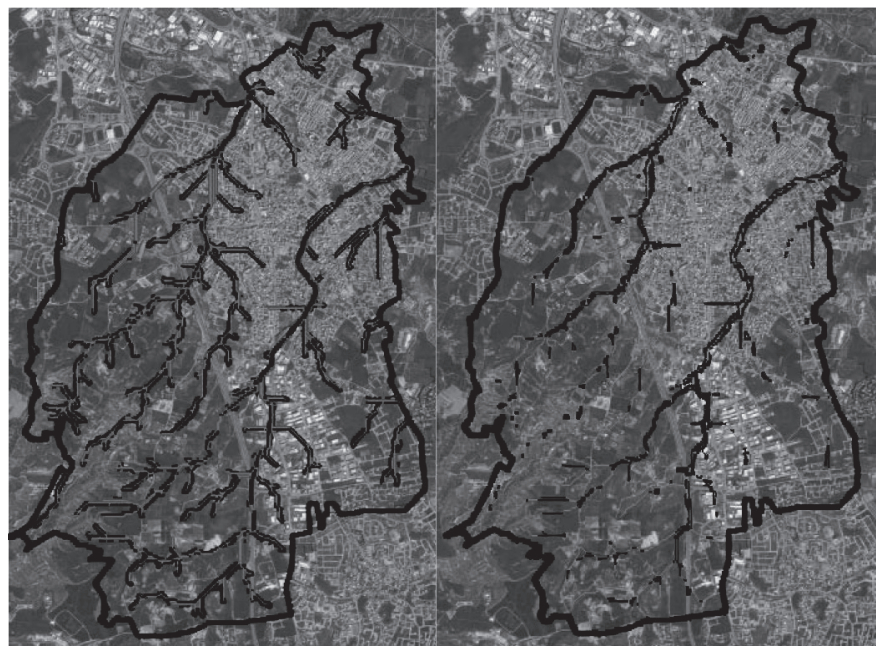

65 I Page

Figure 8: Latsia Hydrological Map and the same map with $10 \mathrm{~m}$ buffer zone (red colour)

\section{Identification of EAPS}

The final map can be produced by overlapping the layers from Fig. 6, 7 and 8. It is possible to identify proper locations for EAPs now, based on the chosen factors. Sixteen polygons, areas (Fig. 9) are finally selected after the rejection of areas with red and yellow colours (not suitable). Calculating geometry in ArcGIS allows us to find the area and perimeter of our polygons (Fig. 10). The size of the selected areas is an essential factor for the final choice and planning. Each polygon should have the proper size in relation to the population. Areas with only one route can be used as secondary alternative locations in cases of limitations considering the space or can be used for other reasons different than facilitating population. 


\section{European Human Journal of Security}

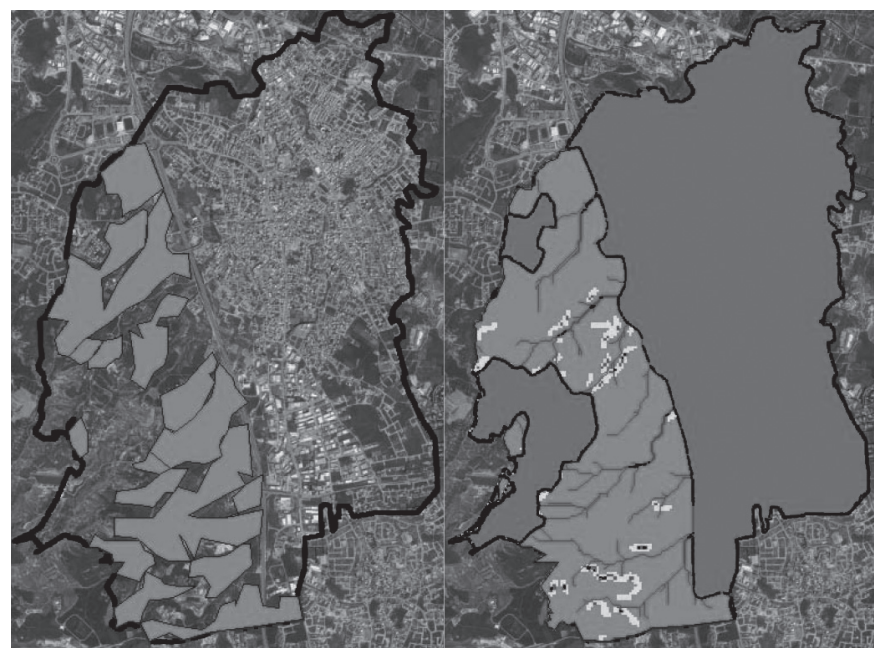

Figure 9: Latsia final map after merging maps from Fig. 6, 7 and 8 (red and yellow colours -no proper locations)

\begin{tabular}{|c|c|c|c|}
\hline \multicolumn{4}{|c|}{ Table } \\
\hline \multicolumn{4}{|c|}{ 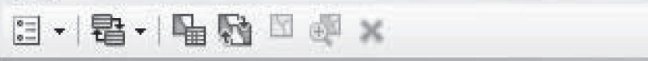 } \\
\hline \multicolumn{4}{|c|}{ EAPsSurface } \\
\hline 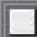 & FID III Shape* & ld & Area $(\mathrm{sg} \mathrm{m})$ \\
\hline E & \begin{tabular}{l|l}
0 & Polygon \\
\end{tabular} & 0 & 248997,53 \\
\hline E & \begin{tabular}{l|l|}
1 & Polygon \\
\end{tabular} & 0 & 858411,57 \\
\hline E & \begin{tabular}{l|l|}
2 & Polygon \\
\end{tabular} & 0 & 203205,66 \\
\hline 口 & \begin{tabular}{l|l|}
3 & Polygon \\
\end{tabular} & 0 & 388418,88 \\
\hline E & \begin{tabular}{l|l}
4 & Polygon \\
\end{tabular} & 0 & 226784,28 \\
\hline E & \begin{tabular}{l|l|}
5 & Polygon \\
\end{tabular} & 0 & 728015,37 \\
\hline E & \begin{tabular}{l|l|}
6 & Polygon \\
\end{tabular} & 0 & 312567,64 \\
\hline$\theta$ & \begin{tabular}{l|l}
7 & Polygon \\
\end{tabular} & 0 & 172211,15 \\
\hline 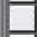 & \begin{tabular}{l|l|}
8 & Polygon \\
\end{tabular} & 0 & 137221,85 \\
\hline & \begin{tabular}{l|l|}
9 & Polygon \\
\end{tabular} & 0 & 38393,59 \\
\hline & 10 Polygon & 0 & 63665,29 \\
\hline 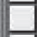 & 11 Polygon & 0 & 45674,84 \\
\hline & \begin{tabular}{l|l}
12 & Polygon \\
\end{tabular} & 0 & 49381,15 \\
\hline & \begin{tabular}{l|l|}
13 & Polygon \\
\end{tabular} & 0 & 119481,88 \\
\hline & 14 Polygon & 0 & 144882,47 \\
\hline & \begin{tabular}{l|l}
15 & Polygon \\
\end{tabular} & 0 & 67310,55 \\
\hline
\end{tabular}

Figure 10: Polygons (evacuation areas) size in square meters

\section{(Area column)}

Examining the existence of a well-established road network and the accessibility to the selected places is the last phase for the identification of proper EAPs locations (Fig. 11). Sites have to be linked with a minimum of two roads/routes. In case of an unexpected incident, areas must have two escape routes to avoid the chance of 


\section{European Human Journal of Security}

population being trapped or must have a straightforward approach to the area using it as an entrance point.

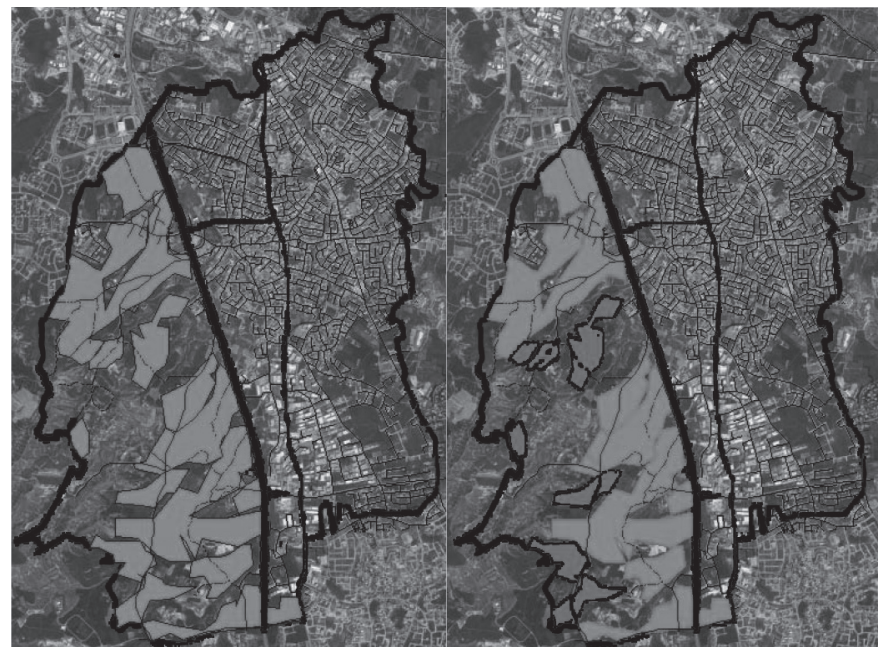

67 I Page

Figure 11: Finally selected polygons and the rejected ones

(in red colour) with only one connecting road

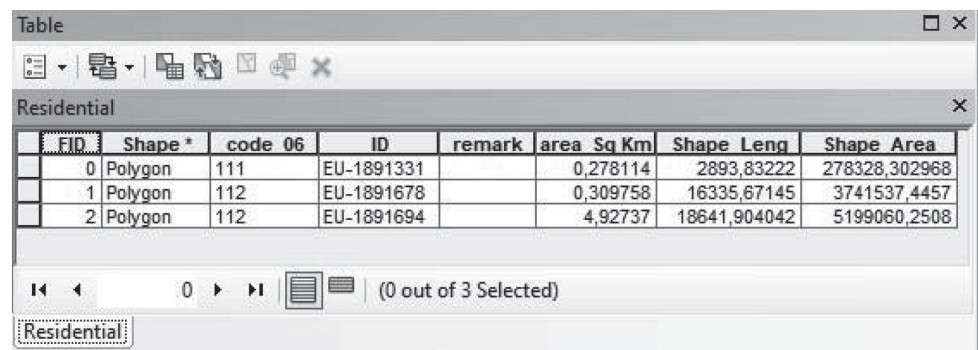

Figure 12: Table of polygons with residential Corine Land Cover codes $(111 \& 112)$

Latsia municipality's total population is 16,774 (Statistical Service, 2019), according to the residential polygons table (Fig. 12), and the area size is $5.52 \mathrm{~km}^{2}$. The total area consists of two different CLC codes, and that means each of them has a different population density. The code area 111 has the size of only $0.28 \mathrm{~km}^{2}$ in total $5.24 \mathrm{~km}^{2}$ and for this reason we can consider that these different codes have the same population density. It is possible to find Latsia's municipality density by calculating the data regarding the area and population sizes. After that, the numbers are put into the population 


\section{European Human Journal of Security}

density formula: Population Density (PD) $=$ Number of People / Land Area. The formula gives us PD = 16774/5.52 $\mathrm{km}^{2}=3.039 \mathrm{per} / \mathrm{km}^{2}$. The residential area will be divided by using the Thiessen tool in equal five parts (Fig. 13).

Page I 68
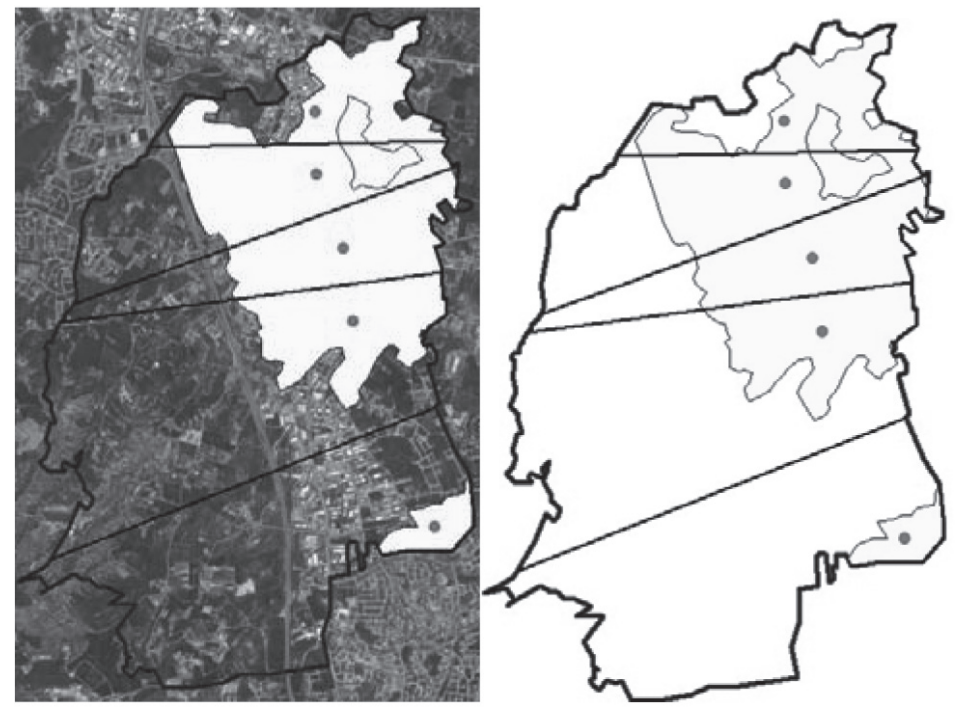

Figure 13: Residential areas (codes 111\&112) divided equally by using the Thiessen tool

Furthermore, in evacuation areas, shelter size in camps and areas per person are vital in the planning phase. Crowded conditions lead to increased morbidity, stress, and other negative emotions/situations. The provision of adequate space, both outside and inside shelters, is an essential requirement. A minimum surface area of $45 \mathrm{~m}^{2}$ per person, including household gardening space, should be allocated. $30 \mathrm{~m}^{2}$ per person will be necessary for roads, footpaths, dining facilities, sanitation, security, firebreaks, administration, water storage, distribution points, storage of relief items, and of course, plots for shelter (UNHCR, 2019). If we calculate the needed area for Latsia municipality population, the result will be $1.258 .050 \mathrm{~m}^{2}\left(45^{*} 16774+30 * 16774\right)$. The selected area size, based on Figure 11 results, is $3.017 .534 \mathrm{~m}^{2}$. In this case, Latsia's population can be evacuated inside the municipality's boundaries as there is more than twice larger area than is required. In the last phase, the physical 


\section{European Human Journal of Security}

environment combined the evacuation areas with the population. In this way, we can succeed in dividing the people to different EAPs by following different routes. EAPs and populations are connected by the same colour of the circle and number (fig. 14).

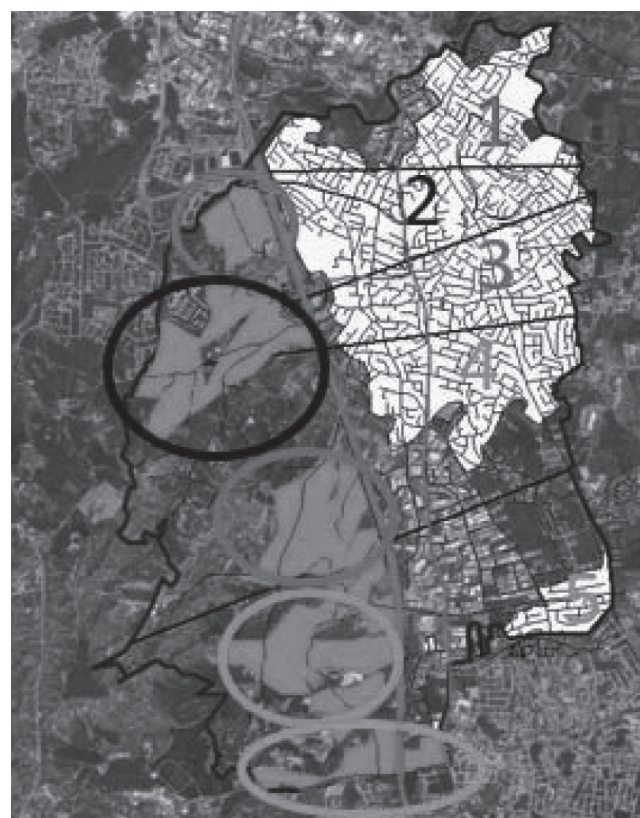

69 I Page

Figure 14: Final map with corresponding residential and evacuation areas combined with the road network

\section{Conclusion and future work}

Nowadays, new technologies such as Geoinformatics, Spatial Analysis, Geographic Information Systems, Data Science, and Analytics provide the required toolbox which is necessary in order to identify and analyse/model the characteristics of each individual, to accomplish the personalisation of his/her security.

A recent unfortunate example of deadly and catastrophic impacts because of the lack of coordination and synchronization between state security stakeholders and the unavailability of reliable information caused the death of 100 persons in a massive wildfire, in July 2018 in the seaside village of Mati, Greece's east coast, not far 


\section{European Human \\ Journal of Security}

from Athens (Greek senior officials charged over deadly wildfires in Mati, 2019). The outcome of the catastrophe could have been different if the responsible public security stakeholders had predetermined the necessary measures in case of emergency EAPs. Furthermore, EAPs could change the outcome even for threats of low probability and high impact (black swan) (Taleb, 2007), for instance, threats like a tsunami incident, in the context of crisis and disaster management, and generally for helping with the very last moment changes in operational plans/strategies.

Additionally, another critical gain from using new technologies is providing the capability/opportunity to an individual to be aware of where they should evacuate based on his/her geospatial profile (specify vulnerabilities and capabilities). At the same time, we become responsible for potential threats (before they become threats). This responsibility can be considered as a starting point for a person to acquire a security mentality. Aftereffect will be the crucial security culture, the resilience of the society, and civil preparedness.

Further use of geospatial profile in order to succeed in personalizing human security will help us take a holistic and more profound approach to the population evacuation process. By the implementation of geospatial profile and personalized security, at the level of municipality, authorities should inform all the residents personally where and how they must arrive at the predetermined EAPs. In this procedure, security stakeholders should take into consideration all the personal and unique characteristics of individuals. (Fig.15), where all the necessary data already exists and is available to the local authorities. The aftereffect of this procedure will be effective and efficient planning, considering the vulnerable population. For instance, security stakeholders in the planning phase can calculate and have buses and other vehicles/means to evacuate pupils, disabled or old people, and different vulnerable categories. For that reason, to succeed in achieving a holistic approach to evacuation planning it is essential for authorities to take into consideration both the human terrain and physical terrain features. 


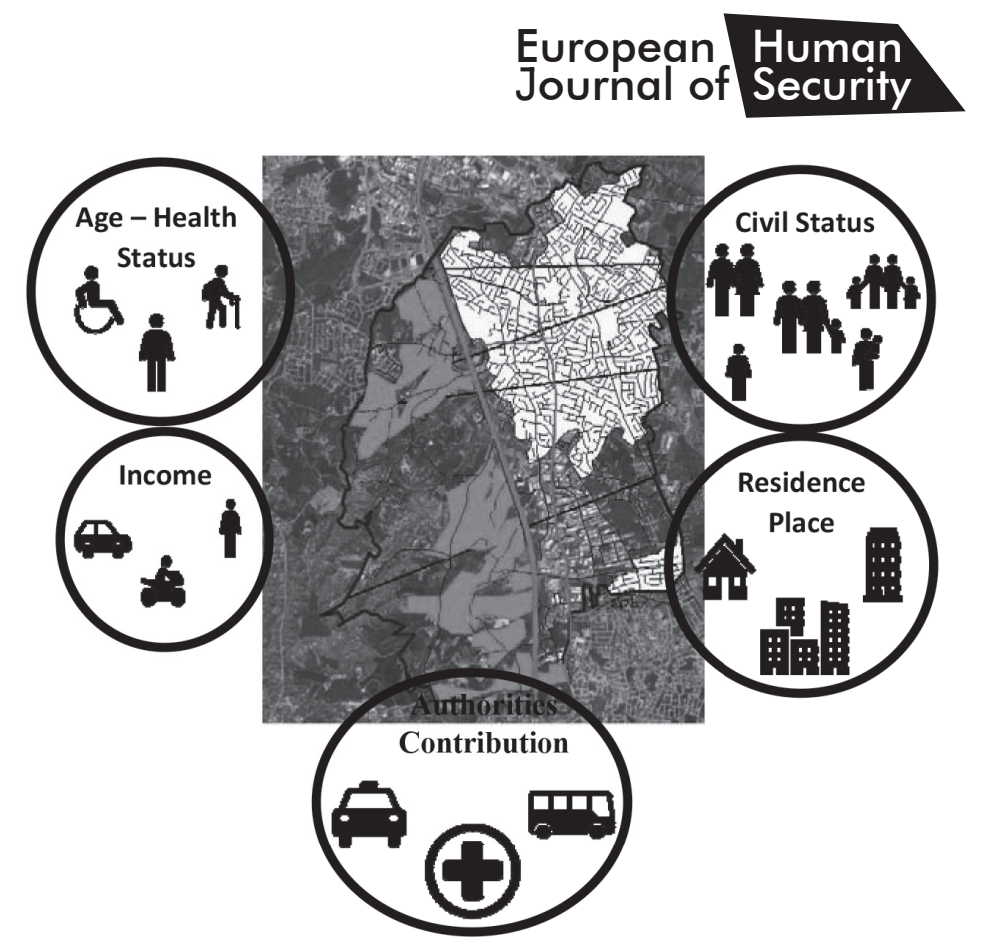

71 I Page

Figure 15: Final map with human-environment dimensions' factors

Personalised human security refers to safety that assesses the geospatial characteristics of each person and applies accordingly to these. Each person has a unique geospatial profile (identity). This identity is spatiotemporal and is formed by the natural (physical) environment in which a person operates and interacts. Moreover, the culture that the person advocates considering its continuously changing nature has the essential role. In order to succeed in personalized functional security, it should be combined with customized information, achieving better results than a mass information approach. Personalized information can be timely, reliable, offer confidence to populations, allow the mitigation of collateral damages in crises, and generally has a positive impact on stakeholders (first responders, policy/decision makers).

Applied human security can act as a bridge connecting academic research with policy-making actors in the field. In this direction, the identification of EAPs provides a very first step/primary tool or method towards security and extending applied human security. 


\section{REFERENCES}

American Association of State Highway and Transportation Officials. (2001). A policy on Geometric Design of Highways and Streets. Washington: AASHTO.

Arru M. \& Negre E. (2018). People behaviors in crisis situations: Three modeling propositions. In T. Comes, F. Bénaben, C. Hanachi, M. Lauras \& A. Montarnal (Eds.), $14^{\text {th }}$ International Conference on Information Systems for Crisis Response and Management: Proceedings of the 14th ISCRAM Conference (pp. 139149), France: Albi.

ASTER Global Digital Elevation Map Announcement. (2004). Retrieved September 25, 2019, from https://asterweb.jpl.nasa.gov/gdem.asp

Chrysoulidis, S.E. \& Kyriakids, P.C. (2018). Personalized security: a step towards applied human security, In S. Stanarević, G. J. Mandić \& LJ. Katić (Eds.), 4th International Conference on Human Security: The Proceedings of Human Security and New Technologies (pp. 107-116), Belgrade: Faculty of Security Studies and Human Security Research Center.

Introduction to SeCure 4: Security Culture. (2018). London: Centre for the Protection of National Infrastructure (CPNI).

CORINE Land Cover. (2018). Retrieved September 25, 2019, from https://land.copernicus.eu/pan-european/corine-land-cover

Greek senior officials charged over deadly wildfires in Mati. (2019). Retrieved September 29, 2019, from https:// www.bbc.com/news/world-europe-47468162

Hansford, B. (2011). Reducing risk of disaster in our communities. Teddington: Tearfund.

Lukacs, M. \& Bhadra, D. (2012). Table of Contents. Schriften des Forschungszentrum Jlich Reihe Energietechnik 21(November), 39. Retrieved from 


\section{European Human Journal of Security}

https://www.ifrc.org/Global/Publications/disasters/vca/Vca_en.pdf

OpenStreetMap (2006). Retrieved September 25, 2019, from https://www.openstreetmap.org/about

Papathoma-Köhle, M. et al. (2007). Elements at Risk as a Framework for Assessing the Vulnerability of Communities to Landslides, Natural Hazards and Earth 73 I Page System Science 7(6): 765-779.

$68 \%$ of the world population projected to live in urban areas by 2050, says UN. (2018). Retrieved October 22, 2019, from www.un.org/development/desa/ en/news/population/2018-revision-of-world-urbanization-prospects.html

Statistical Service. (2019). Republic of Cyprus: Ministry of Finance, Retrieved from https://www.mof.gov. cy/mof/cystat/statistics.nsf/populationcondition

Strahler, A. N. (1957). Quantitative analysis of watershed geomorphology. Eos, Transactions American Geophysical Union, 38(6), 913-920.

Southworth, F. (1991). Regional Evacuation Modelling: A State-of-the-Art Reviewing. Springfield: Oak Ridge National Laboratory.

Taleb, N. N. (2007). The black swan: The impact of the highly improbable. New York: Random house.

UNHCR. (2019). Camp planning standards (planned settlements). Emergency Handbook. Retrieved from https://emergency.unhcr.org/entry/45582/ camp-planning-standards-planned-settlements

UNDP. (2004). Reducing Disaster Risk: A Challenge for Development - a Global Report. Disaster and Crisis Management. Retrieved from http://www.ifrc.org/en/whatwe-do/disaster-management/preparing-for-disaster/risk-reduction/reducing-disaster-risk/

United Nations General Assembly. (2012). Resolution adopted by the General Assembly [without reference to a Main Committee (A/66/L.55/Rev.1 and Add.1)], 66/290 Follow-up to paragraph 143 on human security of the 2005 World Summit Outcome, A/ RES/66/290, 25 October. New York: United Nations. 